\title{
Onze anos de experiência com emprego do anel intraluminal para tratamento das doenças da aorta
}

\author{
Rodrigo de Castro BERNARDES*, Fernando Antônio Roquette REIS FILHO*, Luiz Cláudio Moreira LIMA*, \\ Ernesto Lentz da Silveira MONTEIRO*, José Marcelo Coutinho de MELO*, Sandro Adauto MARTINS*, \\ Geraldo de Rezende PENA*, Pedro Evandro Alvim de FARIA*, Rodrigo Gil GUIMARÃES*, \\ Wanderbilt Duarte de BARROS NETO*, Jefferson Francisco de OLIVEIRA*
}

RBCCV 44205-461

Bernardes R C, Reis Filho F A R, Lima L C M, Monteiro E L S, Melo J M C, Martins S A, Pena G R, Faria P E A, Guimarães R G, Barros Neto W D, Oliveira J F - Onze anos de experiência com emprego do anel intraluminal para tratamento das doenças da aorta. Rev Bras Cir Cardiovasc 1999; 14 (3): 200-6

RESUMO: O tratamento cirúrgico das doenças da aorta é geralmente acompanhado de altas taxas de morbimortalidade. O paciente que tem média de idade avançada geralmente é apresentado ao cirurgião em estado grave, com má condição nutricional, muitas vezes com alterações em vários órgãos e sistemas causados pela própria doença aguda. A correção convencional exige técnicas coadjuvantes complexas e agressivas como a circulação extracorpórea prolongada, hipotermia profunda, parada circulatória total além de prolongados tempos de pinçamento de aorta. Na tentativa de reduzir a agressão cirúrgica no paciente já intensamente debilitado pela própria doença, desenvolvemos, em 1988, um anel intraluminal ${ }^{1,2)}$ que tem medidas projetadas e experimentadas para facilitar a sua manipulação e anastomose, proporcionando uma diminuição acentuada no tempo de operação, tempo de CEC, tempo de pinçamento de aorta, excluindo muitas vezes a circulação extracorpórea e a hipotermia, obtendo uma anastomose fácil, rápida, segura e hemostática. Entre março de 1988 e janeiro de 1999, 432 pacientes foram submetidos a tratamento cirúrgico de dissecções ou aneurismas da aorta em nosso Serviço. Em 328 pacientes empregamos o anel intraluminal como técnica de anastomose. Usamos 489 anéis. Cento e vinte e cinco pacientes eram portadores de dissecção aguda de aorta do tipo A, 29 eram portadores de dissecção aguda de aorta do tipo B, 81 de aneurisma de aorta ascendente, 8 de aneurisma de arco aórtico, 28 pacientes eram portadores de aneurisma de aorta torácica descendente, 17 eram portadores de aneurisma toracoabdominal e 40 pacientes de aneurisma de aorta abdominal infra-renal. A mortalidade global foi de $13,41 \%$. O seguimento ambulatorial destes pacientes variou de 11 anos a 25 dias. A curva actuarial de sobrevivência em 11 anos mostra 67,3\%. Em nenhum caso observamos as complicações descritas na literatura, como embolia, formação de pseudo- aneurisma, roturas ou estenose. Concluindo, em nosso Serviço, o emprego de anel intraluminal facilita o ato cirúrgico, reduz o tempo de anastomose e o sangramento, proporcionando facilidade técnica, redução da mortalidade e boa perspectiva de sobrevida a longo prazo.

DESCRITORES: Aorta, cirurgia. Prótese vascular. Contenedores. Doenças vasculares, cirurgia.

Trabalho realizado no Instituto do Coração do Hospital Madre Teresa. Belo Horizonte, MG, Brasil. Apresentado ao 26ํㅡㄹ Congresso Nacional de Cirurgia Cardíaca. Fortaleza, CE, 8 a 10 de abril, 1999.

* Do Instituto do Coração do Hospital Madre Tereza.

Endereço para correspondência: Rodrigo C. Bernardes. Rua Maranhão, 904 - Apto. 101. Funcionários. Belo Horizonte, MG, Brasil. CEP $30150-330$.

Tel (031)225-8587.Fax (031) 292-0832.e-mail: cecc@bhnet.com.br 
Bernardes R C, Reis Filho F A R, Lima L C M, Monteiro E LS, Melo J M C, Martins S A, Pena G R, Faria P E A, Guimarães R G, Barros Neto W D, Oliveira J F - Onze anos de experiência com emprego do anel intraluminal para tratamento das doenças da aorta. Rev Bras Cir Cardiovasc 1999; 14 (3): 200-6.

\section{INTRODUÇÃO}

O conceito de prótese intraluminal foi introduzido por CARREL (3), em 1912, sugerindo a correção de aneurismas com tubos de vidro e metal. A seguir, HUFNAGEL (4), BLAKEMORE et al. (5) descreveram a técnica em animais. Em 1978, DUREAU et al. (6) e ABLAZA et al. (7) publicaram sua experiência com prótese intraluminal sem sutura. A experiência inicial com a prótese intraluminal apresentou complicações como: trombose e embolia, estenose, roturas e formação de pseudo-aneurisma além de migração da prótese. Estas complicações fizeram com que os grupos cirúrgicos perdessem o entusiasmo e praticamente abandonassem a técnica. Em 1982, LEMOLE et al. (8) publicam uma série de 14 pacientes com ótimos resultados. Em 1988, desenvolvemos o nosso modelo de anel intraluminal, projetando um dispositivo que eliminasse as complicações descritas na literatura, além de poder ser usado em qualquer tipo de prótese vascular, a baixo custo. Após os estudos experimentais, observamos que a prótese intraluminal era eficiente para uma anastomose muito rápida, fácil e segura. Nos estudos em animais, não observamos qualquer complicação como as descritas na literatura. Passamos, então, a empregar a prótese intraluminal para o tratamento cirúrgico das doenças da aorta em 1988. O objetivo deste trabalho é apresentar a nossa experiência com a técnica e avaliar, após 11 anos, de seu emprego os resultados obtidos.

\section{CASUÍSTICA E MÉTODOS}

\section{O Anel Intraluminal}

$\mathrm{O}$ anel tem a propriedade de transformar uma prótese vascular comum de qualquer tamanho, diâmetro, material ou forma em uma prótese intraluminal. $O$ anel tem $25 \mathrm{~mm}$ de comprimento, o que facilita a sua manipulação e posicionamento na luz verdadeira da aorta. $O$ sulco do anel mede $10 \mathrm{~mm}$ de largura e tem $2,5 \mathrm{~mm}$ de profundidade o que nos proporciona muita facilidade de localização e segurança para a ligadura sobre o sulco, mesmo quando o tecido aórtico é espesso, evitando assim migrações ou sangramento.

\section{Casuística}

Entre março de 1988 e janeiro de 1999, 432 pacientes foram submetidos a tratamento cirúrgico das doenças da aorta em nosso Serviço. Em 328 pacientes empregamos o anel intraluminal como técnica de anastomose. Usamos um total de 489 anéis. A média de idade foi de 68,3 anos variando de 18 a 93 anos. O predomínio foi do sexo masculino com 203 pacientes. Cento e vinte cinco pacientes eram portadores de dissecção aguda de aorta do tipo A, 29 pacientes eram portadores de dissecção aguda de aorta do tipo B, 81 pacientes eram portadores de aneurisma de aorta ascendente, 8 eram portadores de aneurisma de arco aórtico, 28 eram portadores de aneurisma de aorta torácica descendente, 17 de aneurisma toracoabdominal e 40 pacientes eram portadores de aneurisma de aorta abdominal infrarenal.

\section{Preparo da Prótese Intraluminal}

O preparo da prótese é feito durante o ato cirúrgico (1). Após a abertura da aorta doente, medimos cuidadosamente a luz verdadeira da aorta com medidores de valvas cardíacas e escolhemos o anel que entre confortavelmente no lume da aorta. Em seguida, a prótese é passada por dentro da luz do anel e invertida por sobre o seu sulco, de modo a vestí-lo, transformando assim esta prótese vascular comum em prótese intraluminal.

\section{Técnica Cirúrgica}

Para se realizar uma anastomose com prótese intraluminal, primeiro examinamos externamente a aorta e escolhemos os prováveis pontos, proximal e distal, onde vamos realizar as anastomoses. Em seguida, com o mínimo de dissecção e manipulação, a aorta é contornada com fios de grosso calibre (Ethibond 5) nos locais escolhidos para a anastomose. Após a heparinização e pinçamento da aorta, realizamos aortotomia com o cuidado de não aproximar muito do pinçamento aórtico. Retiramos os trombos e medimos a luz verdadeira da aorta ou o colo do aneurisma, escolhendo sempre um anel que penetre suavemente na luz aórtica. Preparamos então a prótese intraluminal. Para se posicionar o anel dentro da luz da aorta, pinçamos as bordas laterais do aneurisma, junto ao ponto de anastomose. O auxiliar promove uma tração contrária e em seguida posicionamos a prótese intraluminal e procedemos a ligadura circunferencial extravascular sobre o sulco do anel que por ser largo e profundo é facilmente localizado.

\section{RESULTADOS}

A mortalidade global no pós-operatório imediato foi de $13,41 \%$. Os 44 pacientes que faleceram não tiveram a causa de óbito relacionada diretamente com o emprego de anel intraluminal. A causa mais freqüente de óbito neste grupo de pacientes foi a falência de múltiplos órgãos. Nos pacientes subme- 
Bernardes R C, Reis Filho F A R, Lima L C M, Monteiro E LS, Melo J M C, Martins S A, Pena G R, Faria P E A, Guimarães R G, Barros Neto W D, Oliveira J F - Onze anos de experiência com emprego do anel intraluminal para tratamento das doenças da aorta. Rev Bras Cir Cardiovasc 1999; 14 (3): 200-6.

tidos a tratamento de dissecção aguda de aorta do tipo A, empregando dois anéis, na anastomose proximal e distal (Figuras 1 e 2), o tempo médio de circulação extracorpórea foi de 36 minutos e o tempo médio de pinçamento de aorta foi de 15 minutos. Nos pacientes que apresentavam rotura da intima muito próximo dos óstios coronarianos, empregamos a plastia da raiz da aorta com duplo "patch" e re-suspensão da valva, empregando o anel para a anastomose distal. Nestes casos o tempo médio de CEC foi de 66 minutos e o pinçamento médio de aorta foi de 34 minutos. Somente em 15\% dos pacientes substituímos a valva aórtica, empregando tubo valvado pelas técnicas de Bentall-DeBonno (1 paciente desenvolveu BAVT definitivo) ou Cabrol. A média de reposição de sangue foi de $710 \mathrm{ml} /$ paciente. Somente $2,5 \%$ dos pacientes retornaram ao bloco cirúrgico para revisão da hemostasia. O tempo médio de ventilação mecânica foi de 11,7 horas e de permanência no CTI foi de 4,4 dias. Os 28 pacientes submetidos a aneurismectomia de aorta descenden-

Fig. 1 - Tratamento cirúrgico da dissecção aguda de aorta do tipo A. Preparo da prótese intraluminal com dois anéis.

Fig. 2 - Aspecto cirúrgico anel intraluminal proximal anastomosado acima dos óstios coronarianos. Anel distal preparado para implante. te tiveram um tempo médio de pinçamento de aorta de 8 minutos e somente tivemos $1(3,5 \%)$ caso de paraplegia. Nos 17 pacientes submetidos a tratamento de aneurisma toracoabdominal, empregamos o anel na anastomose proximal quando possível e sempre na anastomose distal, reduzindo o tempo de pinçamento de aorta e a isquemia visceral e medular. Tivemos $1(5,8 \%)$ casos de paraparesia, com recuperação total no $5^{\circ}$ dia. No seguimento tardio destes pacientes que variou de 130 meses a 25 dias não observamos nenhuma complicação diretamente relacionada ao emprego do anel intraluminal. Dois pacientes que haviam sido operados na fase aguda de dissecção aguda de aorta do tipo A, empregando dois anéis, desenvolveram no pós-operatório tardio insuficiência aórtica valvar grave por dilatação da aorta. Um paciente evoluiu com endocardite do tubo valvado tardiamente e dois pacientes apresentaram infecção de prótese anastomosada em aorta descendente tardiamente, evoluindo para o óbito. A curva actuarial de sobrevivência dos pacientes operados com prótese intraluminal mostra uma sobrevivência de $67,3 \%$ em 11 anos.

\section{COMENTÁRIOS}

A prótese intraluminal sem sutura foi introduzida para anastomose em operações da aorta há 20 anos $(6,7)$, acenando ser a melhor alternativa técnica para a realização de uma operação rápida e segura. Porém, durante a fase inicial da experiência, complicações foram descritas como migração, formação de pseudo-aneurisma, hemólise e estenose ao nível da anastomose ${ }^{(9)}$. Em nossa opinião, estas complicações estão relacionadas com o tipo de anel usado nas primeiras anastomoses. Estes anéis eram curtos e apresentavam um sulco pouco profundo o que dificultava o seu posicionamento e ligadura sob 0 tecido espesso da aorta doente, levando a ligaduras pouco eficientes e, conseqüentemente, a migrações e roturas. Em publicações recentes $(10,11)$, o grupo de Lemole refere, que o maior problema das próteses intraluminais resulta do material protético ultrapassado empregado em sua confecção. O anel intraluminal usado por nós pode ser empregado com qualquer tipo de prótese, devendo ser preparado durante a operação. O desenho do anel permite uma fácil anastomose, sendo impossível após a ligadura haver migração da prótese. Quando abordamos um paciente com dissecção aguda de aorta do tipo A, temos a preocupação de oferecer um diagnóstico rápido e preciso, tratamento cirúrgico imediato e abordagem o menos agressiva possível, visando com isto alcançar o melhor resultado (12). Todos os pacientes são operados com circulação extracorpórea em normotemia. Após o pinçamento da aorta empregamos 
Bernardes R C, Reis Filho F A R, Lima L C M, Monteiro E LS, Melo J M C, Martins S A, Pena G R, Faria P E A, Guimarães R G, Barros Neto W D, Oliveira J F - Onze anos de experiência com emprego do anel intraluminal para tratamento das doenças da aorta. Rev Bras Cir Cardiovasc 1999; 14 (3): 200-6.

cardioplegia gelada direto nos óstios coronários. Fazemos, então, uma curta parada circulatória (em normotermia) e observamos o arco aórtico. Somente abordamos o arco aórtico quando existe rotura ou laceração da intima neste ponto onde empregamos a hipotermia a $18^{\circ} \mathrm{C}$ (somente em $12,5 \%$ dos casos). A insuficiência da valva aórtica (moderada/ grave) estava presente em $57 \%$ de nossos pacientes e somente em $15 \%$ deles não conseguimos consersar a valva através de plastia da aorta ou resuspensão devolvendo o suporte perdido pela ligadura do anel proximal. O tempo médio de circulação extracorpórea e de pinçamento de aorta foi muito reduzido em nossos casos decorrente da facilidade e simplicidade da anastomose com anel intraluminal. Com esta abordagem pouco agressiva, conseguimos reduzir a mortalidade para $12,5 \%$. Nos pacientes portadores de dissecção aguda de aorta do tipo $B$, somente indicamos operação quando o paciente apresenta complicações graves durante a sua evolução. Este conceito antigo, esta sendo substituído na medida em que observamos a mortalidade imediata dos pacientes tratados clinicamente, somados à mortalidade tardia e as graves complicações como os grandes aneurismas toracoabdominais desenvolvidos a médio prazo. Quando o paciente não apresenta rotura da aorta, preferimos evitar a manipulação do tecido aórtico doente e friável, operando pela técnica da Tromba de Elefante descrita por BORST et al. ${ }^{(13)}$ adaptada por BUFFOLO \& PALMA ${ }^{(14)}$ e modificada por nós ${ }^{(15)}$ com o emprego do anel intraluminal (Figura 3). Operamos estes pacientes em hipotermia leve, $25^{\circ} \mathrm{C}$. Uma curta parada circulatória é suficiente para a abertura do arco aórtico e posicionamento da prótese em tromba com anel proximal. O tempo médio de parada circulatória foi de $5 \mathrm{~min}$. Operamos 17 pacientes e em 3 deles tivemos complicações com o posicionamento da prótese em aorta descendente. Dois pacientes faleceram e 1 paciente foi reoperado por toracotomia, com sucesso. Somente um dos pacientes submetidos à operação pela técnica da tromba de elefante, manteve o fluxo na falsa luz com dilatação sendo submetido a complementação do tratamento cirúrgico por toracotomia. Os 12 pacientes restantes foram operados por abordagem direta da aorta descendente com 3 óbitos. Dois pacientes portadores de dissecção aguda de aorta tipo B que foram operados devido a paraplegia, como complicação pré-operatória, tiveram recuperação total dos movimentos no pós-operatório (Figura $4 \mathrm{e}$ 5). Nos pacientes portadores de aneurismas de aorta ascendente empregamos 0 anel na anastomose distal para reduzir o sangramento e o tempo de pinçamento de aorta. Nos pacientes com aneurisma de arco aórtico empregamos o anel nas anastomoses proximal e distal fazendo a anastomose convencional

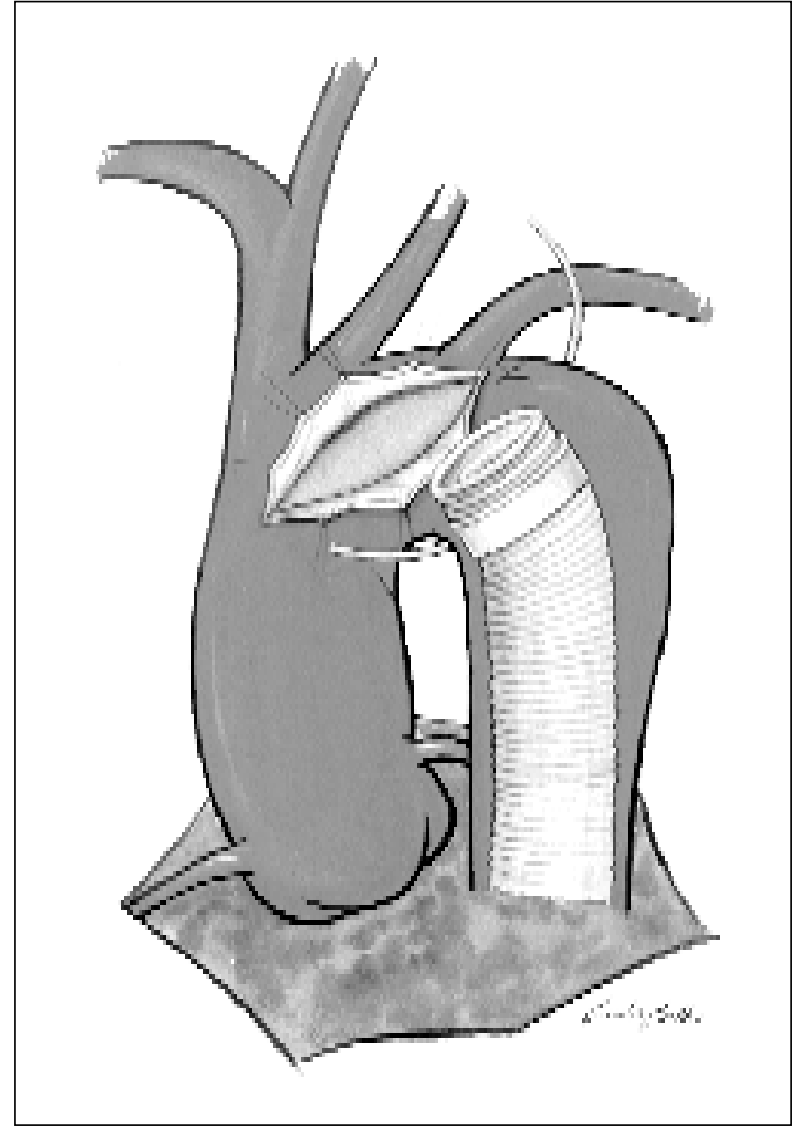

Fig. 3 - Técnica da "tromba de elefante" modificada pelo emprego do anel intraluminal.

dos vasos da base, diminuindo o tempo de parada circulatória total. Em todos os pacientes submetidos a tratamento de aneurisma ou dissecções da aorta descendente, torácica, toracoabdominal e abdominal, não empregamos nenhuma técnica descrita de proteção medular e tivemos apenas um caso de paraplegia o que corresponde a $1,7 \%$. Isto talvez se deva ao reduzido tempo de isquemia medular. Uma das mais interessantes aplicações do anel intraluminal é o tratamento do aneurisma toracoabdominal roto (Figuras 6, 7 e 8), abordando somente o segmento roto da aorta (16). A anastomose com anel intraluminal no tecido aneurismático é fácil e segura oferecendo a oportunidade ao paciente de um tratamento muito pouco agressivo, evitando a toracofrenolaparotomia, tempo prolongado de isquemia visceral e sangramento durante a fase aguda, deixando o tratamento definitivo do resto da aorta aneurismática para um segundo tempo, com o paciente estável, em boas condições clínicas. Outra interessante aplicação da prótese intraluminal foi descrita por SADAHIRO et al. (17) na correção de aneurismas com aorta calcificada. No seguimento a longo prazo destes pacientes que variou de 11 anos a 25 dias, 
Bernardes R C, Reis Filho F A R, Lima L C M, Monteiro E LS, Melo J M C, Martins S A, Pena G R, Faria P E A, Guimarães R G, Barros Neto W D, Oliveira J F - Onze anos de experiência com emprego do anel intraluminal para tratamento das doenças da aorta. Rev Bras Cir Cardiovasc 1999; 14 (3): 200-6.

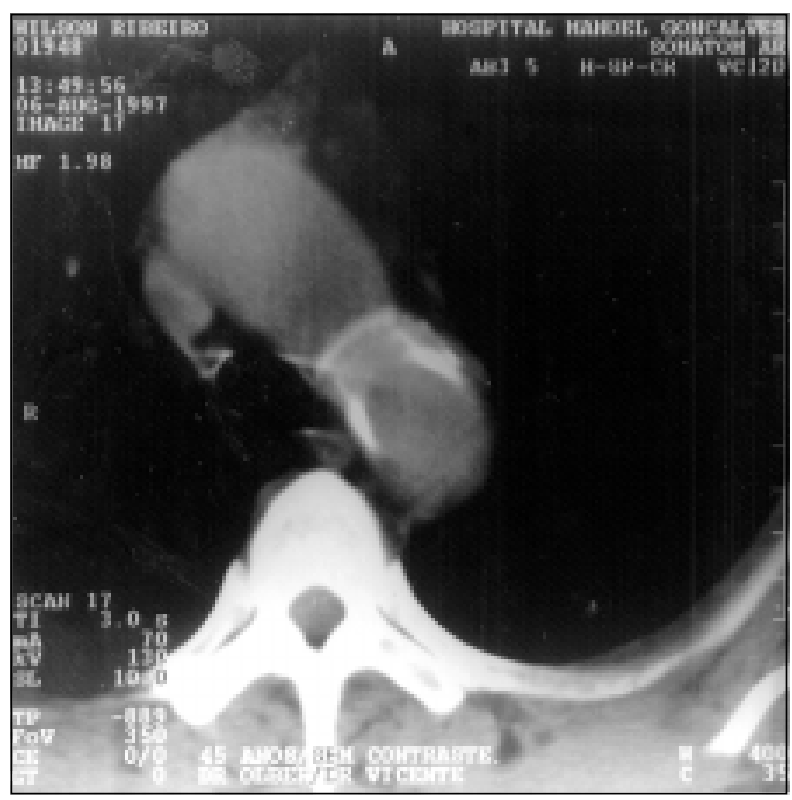

Fig. 4 - Controle tomográfico tardio de paciente submetido à correção de dissecção aguda de aorta do tipo B. Observar anel intraluminal após a origem de subclávia esquerda.

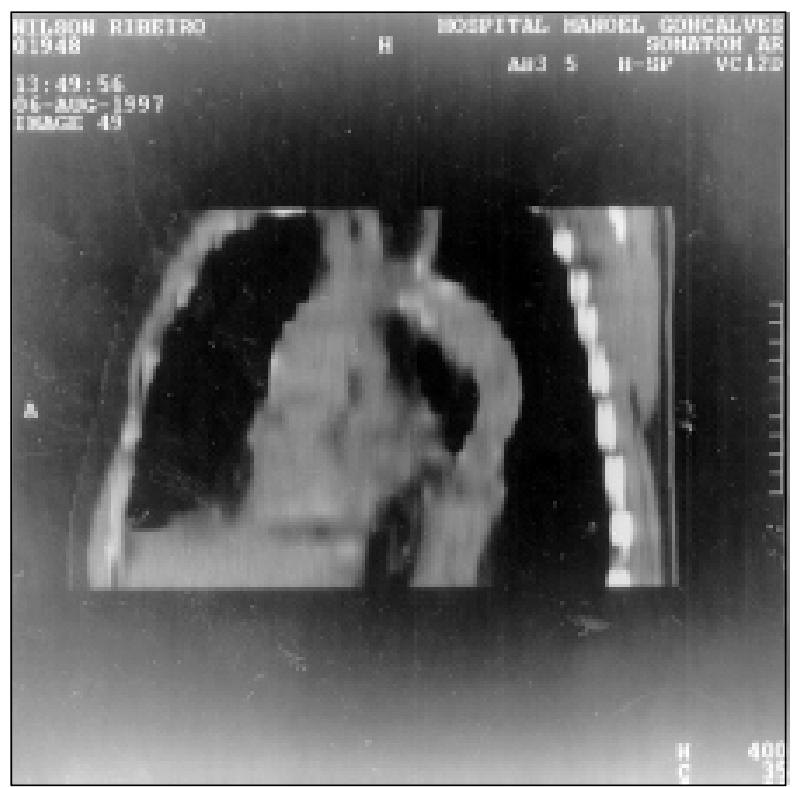

Fig. 5 - Controle tomográfico tardio de paciente submetido à correção de dissecção aguda de aorta do tipo B pela técnica da "tromba de elefante". Observar regressão da falsa luz e recanalização total da aorta distal.

média de 49 meses, não observamos qualquer complicação referente ao uso do anel intraluminal a curto e longo prazos. Três pacientes evoluíram no pósoperatório tardio com infecção de prótese, vindo a falecer. Dois pacientes submetidos a tratamento de dissecção aguda da aorta do tipo $A$, empregando dois anéis, desenvolveram no pós-operatório tardio,

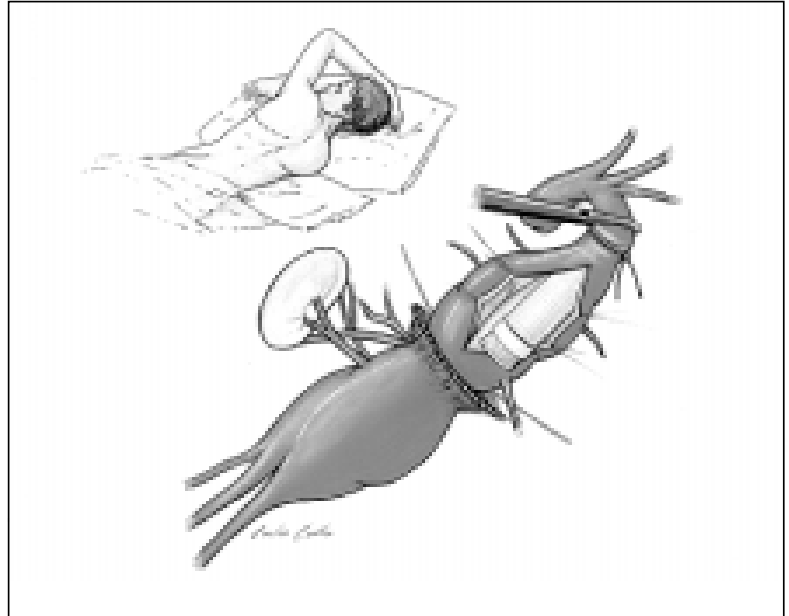

Fig. 6 - Tratamento cirúrgico do aneurisma toracoabdominal em dois tempos. Abertura somente do segmento roto do aneurisma.

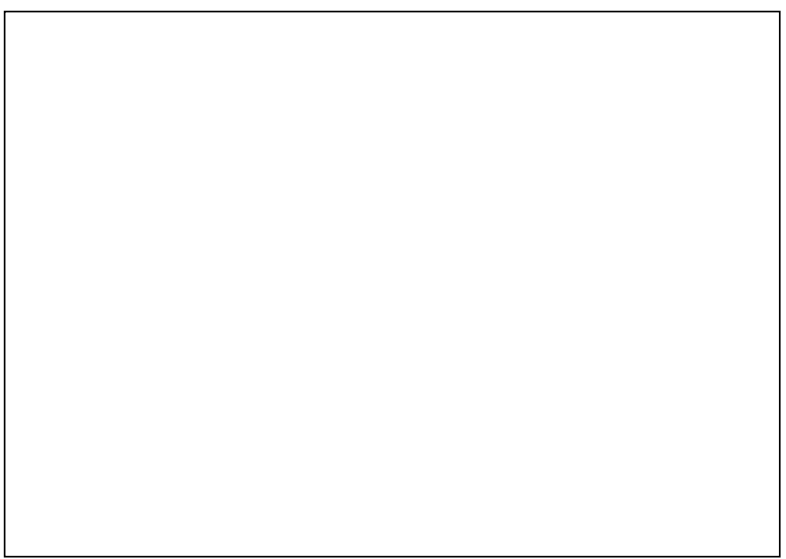

Fig. 7 - Anastomose com anel intraluminal em tecido aórtico aneurismático. Detalhe cirúrgico.

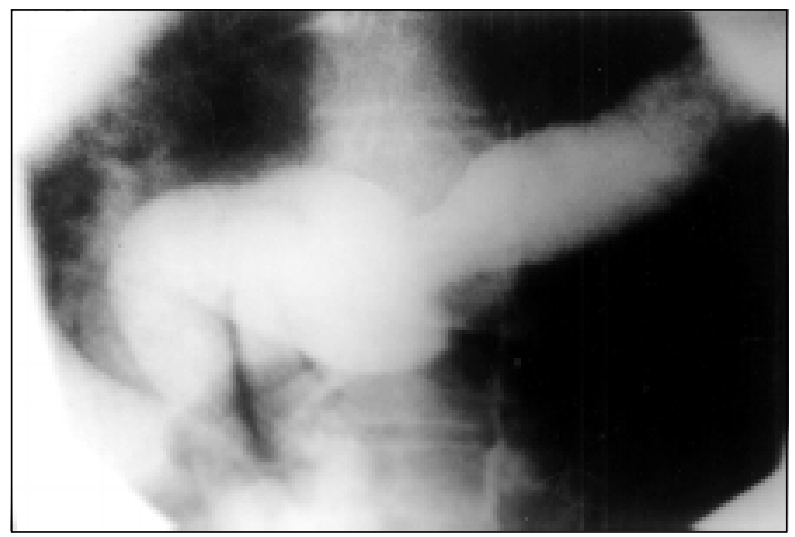

Fig. 8 - Controle arteriográfico tardio do paciente anterior, tratado somente a porção torácica de grande aneurisma dissecante toracoabdominal roto. 
Bernardes R C, Reis Filho F A R, Lima L C M, Monteiro E LS, Melo J M C, Martins S A, Pena G R, Faria P E A, Guimarães R G, Barros Neto W D, Oliveira J F - Onze anos de experiência com emprego do anel intraluminal para tratamento das doenças da aorta. Rev Bras Cir Cardiovasc 1999; 14 (3): 200-6.

insuficiência aórtica valvar por dilatação da aorta. Durante a reoperação, encontramos os anéis completamente incorporados à aorta, com acentuada fibrose sobre o ponto de ligadura. Nos 2 casos, substituímos a valva aórtica e início da aorta, empregando um tubo valvado, anastomosando-o em seguida na antiga prótese intraluminal, conservando a anastomose distal intraluminal prévia. Um dos pacientes submetidos à correção de dissecção aguda de aorta do tipo B manteve a falsa luz com dilatação e foi reoperado após três anos. Feita toracotomia esquerda e somente anastomosamos a extremidade distal da "tromba de elefante" empregando um anel de $24 \mathrm{~mm}$ em sua porção distal.

\section{CONCLUSÃO}

O emprego do anel intraluminal proporciona uma anastomose hemostática, fácil, rápida, e segura, podendo ser realizada mesmo em centros com poucos recursos. A anastomose com prótese intraluminal mostrou ser uma técnica eficiente, pouco agressiva, reduzindo o tempo cirúrgico, proporcionando facilidade técnica, redução da mortalidade e boa perspectiva de sobrevida a longo prazo. Deve portanto fazer parte do arsenal terapêutico de todos os cirurgiões cardiovasculares para ser empregada em casos selecionados.

RBCCV 44205-461

Bernardes R C, Reis Filho F A R, Lima L C M, Monteiro E L S, Melo J M C, Martins S A, Pena G R, Faria P E A, Guimarães R G, Barros Neto W D, Oliveira J F - Use of the intraluminal aortic ring for treatment of aortic diseases: an eleven year experience. Rev Bras Cir Cardiovasc 1999; 14 (3): 200-6

ABSTRACT: The surgical management of diseases of the aorta is usually followed by high rates of morbidity/mortality. The patient who is usually elderly and debilitated presents in serious condition, and often with changes in various organs and systems caused by the acute disease itself. Conventional surgery requires complex coadjuvant techniques such as prolonged extracorporeal circulation (ECC), deep hypothermia, complete circulatory arrest besides prolonged time of aortic clamping. In 1988, in an attempt to reduce the surgical aggression on this already feeble patient, we developed an intraluminal ring ${ }^{(1,2)}$. It was projected and measurements were experimented to simplify its handing and anastomosis resulting in a marked decrease in operative time, ECC time and aortic clamping. It often eliminated the need of ECC and hypothermia resulting in easy, rapid, safe and hemostatic anastomosis. Between March 1988 and Jannuary 1999, 432 patients underwent surgical treatment of dissections or aneurysms of the aorta on our Service. The intraluminal ring with anastomosis was employed in 328 patients. Four hundred and eighty-nine rings were utilized. Diseases included acute dissection of the aorta type $A(25)$, acute dissection of the aorta type $B(29)$, aneurysms of the ascending aorta (81), aneurysms of the aortic arch (08), aneurysms of the descending thoracic aorta (28), thoraco-abdominal aneurysms (17) and aneurysms of the infra-renal abdominal aorta (40). Over-all mortality was $13.41 \%$. Out-patient followup ranged from 11 years to 25 days. The actuarial survival curve in 11 years was $67.3 \%$. In no case did we observe the complications described in the literature such as emboli, formation of pseudo-aneurysms, ruptures or stenosis. On our Service, use of the intraluminal ring simplifies surgery, shortens the time necessary for anastomosis and reduces bleeding, simplifying techniques, reducing mortality and providing a good perspective of survival over the long term.

DESCRIPTORS: Aorta, surgery. Blood vessel prosthesis. Stents. Vascular diseases, surgery.

\section{REFERÊNCIAS BIBLIOGRÁFICAS}

1 Bernardes R C, Reis F A R, Rabelo R C et al. Correção cirúrgica dos aneurismas da aorta: novo dispositivo que transforma qualquer tipo de prótese intraluminal. Rev Bras Cir Cardiovasc 1994; 9: 54-9.

2 Bernardes R C, Reis Filho F A, Marino R L et al. Surgical correction of aortic disease using intraluminal, crimped bovine pericardial graft. Ann Thorac Surg 1995; 60: s 316-21.
3 Carrel A - Results of the permanent intubation of the thoracic aorta. Surg Gynecol Obstet 1912; 15: 245-8.

4 Hufnagel A H - Aortic plastic valvular prosthesis. Bull Georgetown Univ Med Ctr 1951; 4: 128-9.

5 Blakemore A H, Lord J W, Stefco P L - The severed primary artery in the war wounded. Surgery $1942 ; 12$ : 488-508.

6 Dureau G, Villard J, George M, Deliry P, Froment J C, Clermont A - New surgical technique for the operative management of acute dissections of the ascending 
Bernardes R C, Reis Filho F A R, Lima L C M, Monteiro E LS, Melo J M C, Martins S A, Pena G R, Faria P E A, Guimarães R G, Barros Neto W D, Oliveira J F - Onze anos de experiência com emprego do anel intraluminal para tratamento das doenças da aorta. Rev Bras Cir Cardiovasc 1999; 14 (3): 200-6.

aorta: report of two cases. J Thorac Cardiovasc Surg 1978; 76: 385-9.

7 Ablaza S G, Ghosh S C, Grana V P - Use of a ringed intraluminal graft in the surgical treatment of dissecting aneurysms of the aorta: a new technique. $J$ Thorac Cardiovasc Surg 1978; 76: 390-6.

8 Lemole G M, Strong M D, Spagna P M, Karmilowicz N $P$ - Improved results for dissecting aneurysms: intraluminal sutureless prosthesis. J Thoracic Cardiovasc Surg 1982; 83: 249-55

9 Lemole G M - Aortic replacement with sutureless intraluminal grafts. Tex Heart Inst 1990; 17: 302-9.

10 Oz M C, Ashton R C Jr, Mc Nicholas K W, Lemole G M - Sutureless ring graft replacement of ascending aorta and aortic arch. Ann Thorac Surg 1990; 50: 74-9

11 Oz M C, Ashton R C Jr, Lemole G M - As originally published in 1990: Sutureless ring graft replacement of ascending aorta and aortic arch: updated in 1998. Ann Thorac Surg 1998; 65: 1186-7.

12 Bernardes R C - Dissecção aguda de aorta. Tratamento cirúrgico precoce com menor agressividade: maior chance de sobrevivência. Rev Soc Bras Terap Intens 1996; 8: 68-74.

13 Borst H G, Walterbusch G, Schaps D - Extensive aortic replacement using "elephant trunk" prosthesis. Thoracic Cardiovasc Surg 1983; 31: 37-40.

14 Buffolo E \& Palma H - "Tromba de elefante" modificada nas dissecções de aorta. Rev Soc Cardiol Estado de São Paulo 1994; 5: 467-82.

15 Bernardes R C, Rabelo R C, Reis F A R et al. - Tratamento cirúrgico das dissecções agudas de aorta do tipo B. Técnica da tromba de elefante modificada pelo emprego de prótese intraluminal sem sutura. Rev Bras Cir Cardiovasc 1996; 11: 12-7.

16 Bernardes R C, Reis F A R, Lima L C M et al. Tratamento cirúrgico do aneurisma tóraco-abdominal rôto em dois tempos empregando prótese intraluminal sem sutura. Rev Bras Cir Cardiovasc 1997; 13: $41-6$

17 Sadahiro M, Oda K, Togo T, Ohmi M, Tabayashi K Sutureless ringed graft replacement of descending thoracic aorta with extensive calcification. Ann Thorac Cardiovasc Surg 1998; 4: 96-8. 